\title{
Nonquantized Frequency-Modulated Altimeter
}

\author{
Henry P. Kalmus, John C. Cacheris, and Herbert A. Dropkin
}

\begin{abstract}
A new principle that makes possible nonquantized altitude information so that the accurate range of altimeters can be extended to include low-level operation is described. A frequency shifter is inserted between the local oscillator and the mixer in order to remove the inherent error in conventional altimeters that makes them objectionable for low-altitude operation. The authors show how the characteristic of the amplifier can be shaped differently and point out that the strength of the return signal and the inherent amplitude modulation determine the most suitable characteristic for any particular operating condition.
\end{abstract}

\section{Introduction}

In certain altimeters based on continuous-wave operation, a frequency-modulated wave is transmitted toward the ground. The reflected wave has a time delay dependent on distance so that it is possible to measure altitude by comparing the transmitted with the reflected wave. Any modulation function can be employed. A triangular function is more advantageous for special cases. The following analysis, however, is based on sinusoidal modulation. Conventional altimeters are analyzed first.

The transmitted wave is defined by the equation

$$
E_{T}=A \cos \left(\omega_{c} t+\beta \sin \omega_{r} t\right)
$$

where $A$ is the maximum carrier amplitude; $\omega_{c}$ is $2 \pi f_{c}$ equal to $2 \pi\left(c / \lambda_{c}\right)$, where $f_{c}$ is the average carrier frequency; $\beta$ is $F / f_{r}$, where $F$ is the maximum frequency deviation and $f_{r}$ the modulating frequency; $\beta$ is the maximum phase excursion, sometimes called the modulation index; and $\omega_{r}$ is $2 \pi f_{r}$.

The equation for the reflected wave is

$$
E_{R}=K A \cos \left[\omega_{c}(t-\tau)+\beta \sin \omega_{\tau}(t-\tau)\right]
$$

where $K$ is a factor smaller than one that depends on space attenuation, surface-reflection coefficient, antenna gain, etc; $\tau$ is the transit time; and $\tau$ is $2 \mathrm{D} / \mathrm{c}$, where $D$ is the altitude and $c$ the velocity of light. $E_{T}$ and $E_{R}$ are fed into a mixer so that, if $E_{T}>>E_{R}$, the detected signal becomes $E_{c}=K A \cos \phi$, where $\phi$ is the phase angle between $E_{T}$ and $E_{R}$. An expression for $\phi$ is obtained by subtracting the phase angle of $E_{R}$ from the phase angle of $E_{T}$.

$$
\begin{aligned}
& \phi=\omega_{c} \tau+\beta\left[\sin \omega_{r} t-\sin \omega_{r}(t-\tau)\right] \\
& \phi=\omega_{c} \tau+2 \beta \cos \frac{\omega_{r}(2 t-\tau)}{2} \sin \frac{\omega_{\tau} \tau}{2} \\
& \phi=\omega_{c} \tau+\gamma \cos \omega_{r}\left(t-\frac{\tau}{2}\right)
\end{aligned}
$$

where $\gamma=\mathrm{a}$ distance-dependent and time-independent phase angle $\gamma=2 \beta \sin \left[\left(\omega_{r} \tau\right) / 2\right]$. Thus

$$
E_{c}=K A \cos \left[\omega_{c} \tau+\gamma \cos \omega_{\tau}\left(t-\frac{\tau}{2}\right)\right] .
$$

If $\left(\omega_{r} \tau\right) / 2$ is small,

$$
\begin{aligned}
& \gamma \cong \frac{2 F}{f_{r}} \cdot \frac{2 \pi f_{r} \cdot 2 D}{2 c} \\
& \gamma \cong \frac{4 \pi F D}{c}
\end{aligned}
$$

The received signal as described by eq (3) is shown in figure 1. At the left, the received signal is represented as vector $K A$. It swings back and forth by the maximum phase excursion $\gamma$ during each cycle of the modulating frequency $f_{r}$. Its mean position depends on the phase of the reflected carrier wave. If the distance is changed by $\lambda_{c} / 4$, the mean position changes by $180^{\circ}$. It is assumed that $F \ll f_{c}$ so that, for this distance variation, $\gamma$ is essentially constant. At the right, the projection of this vector on the local vector $A$ is shown. Hence, the right side of the figure represents the detected signal. Although, for the case shown, $\gamma$ is only $\pi / 6$, in a general case, $\gamma$ can be a considerable number of full rotations so that a number of complete cycles appear as shown in figure 2. The instantaneous frequency of this wave changes sinusoidally because the angular velocity of vector $K A$ follows a sine function. At the mean position of the vector $K A$ between maximum phase excursions, the angular velocity and, hence, the instantaneous frequency, reach a maximum. If triangular modulation were used, a constant instantaneous frequency with phase reversals would be observed.

The distance information is, as already stated, contained in $\gamma$. In conventional altimeters, the number of zero-crossings per repetition cycle in figure 2 is measured so that the value of $\gamma$ can be determined. Let us assume that for a very close distance, the vector $K A$ has a mean position that is horizontal. Corresponding to the assumed small transit time, $\gamma$ has a small value so that a count of 1 per repetition cycle $(N=1)$ is obtained (fig. 3 ). If the distance is increased by $\lambda_{c} / 8$, the average position of vector $K A$ becomes vertical as shown in figure 4 . A count of 2 per repetition period is now observed. After an additional increase of distance by $\lambda_{c} / 8$, a count of 1 will be obtained again. If this analysis is carried out for larger and larger distances, a graph is obtained for the counts as a function of distance as shown in figure 5 . 


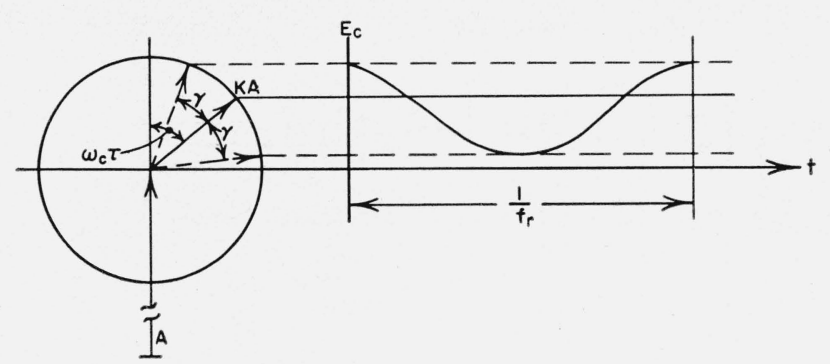

Figure 1. Vector diagram and waveform of the detected signal.

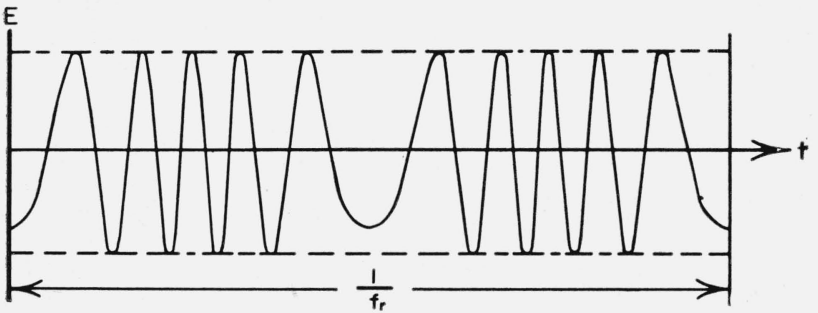

Figure 2. Detected signal waveform for large $\gamma$

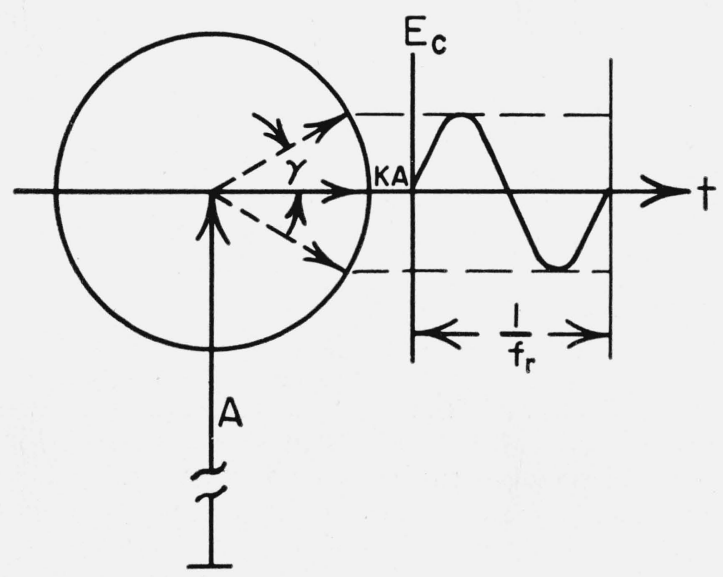

Figure 3. Detected signal producing one count $(\gamma=\pi / 6)$.

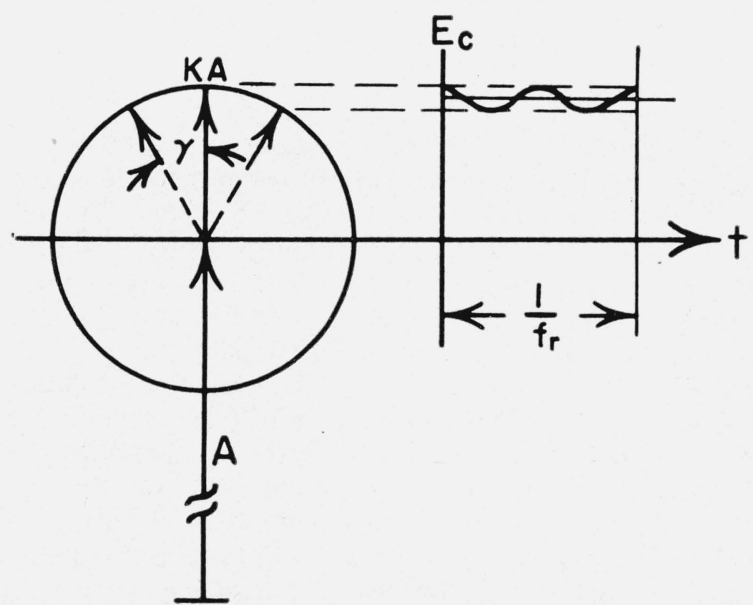

Figure 4. Detected signal producing two counts $(\gamma=\pi / 6)$.

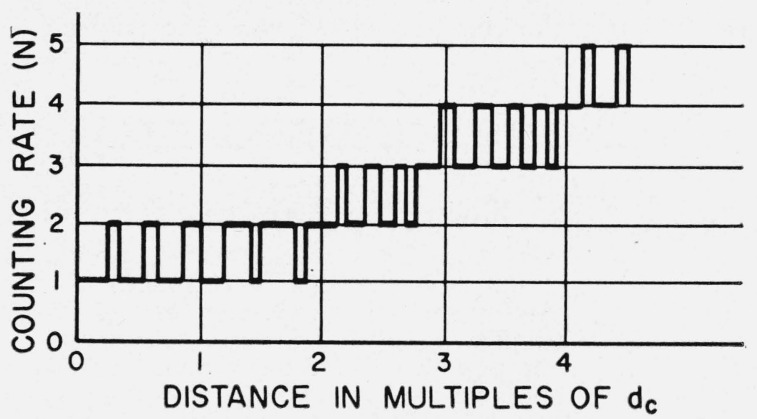

FiguRE 5. Counting rate versus distance for conventional altimeter.
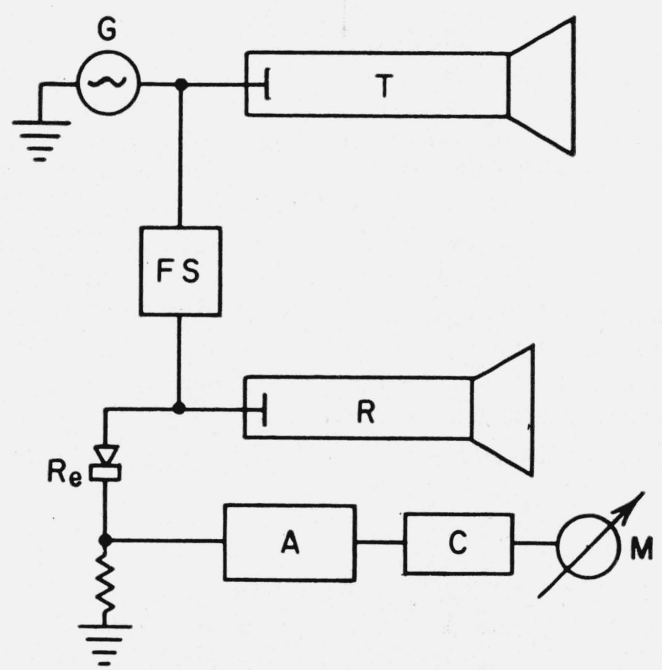

Figure 6. Block diagram of nonquantized altimeter.

Distance $d_{c}$ is called the critical distance. Its value is determined by the fact that $\gamma$ becomes $\pi / 2$ for $d_{c}$ and $n(\pi / 2)$ for $n d_{c}$. Another definition that permits the measurement of $d_{c}$ by counters only is suggested: The critical distance for any $\gamma$ greater than $\pi$ is the largest distance within which the count fluctuates between two distinct values $n$ and $n+1$. Hence, if in a conventional altimeter a certain count, for example, 3 per repetition period, is observed, the distance is indeterminate within the limits between $2 d_{c}$ and $4 d_{c}$. This indeterminacy is usually reduced by the use of a large frequency deviation so that $d_{c}$ becomes small. Furthermore, some averaging effect may be obtained due to the roughness of the terrain, which produces a random variation of height as measured from an aircraft with a horizontal flight path.

The new instrument, which is described in this report, makes it possible to obtain nonquantized distance information using small frequency deviations and over smooth terrain. Heights less than the critical distance can be measured with accuracy. Figure 6 is a block diagram of the new instrument. $\mathrm{G}$ is a frequency-modulated microwave generator coupled to the transmitting antenna T. A small part of the signal produced by $\mathrm{G}$ is fed through the frequency shifter FS and combined with the signal received by the receiving antenna $R$. The frequency 
shifter is either a mechanical or electrical device producing a single-sideband component. Devices of this kind are described in the Appendix. FS has to change the frequency $f_{c}$ of an incoming signal into $f_{c}+f_{d}$, where $f_{d}$ is much smaller than $f_{c}$. A small amount of energy at frequency $f_{r}$ will appear in the output of the frequency shifter, but it is easy to keep the undesired voltage down to 10 percent of the desired signal voltage. The combined signals are rectified to the rectifier $R_{e}$, amplified in the amplifier $\mathrm{A}$, and fed into an averaging counter $\mathrm{C}$, which counts positive-going zero-crossings. Meter $\mathrm{M}$ shows the number of zero-crossings per second.

As already stated, the ambiguity of conventional altimeters is due to the fact that whenever the distance is varied by $\lambda_{c} / 8$, the count is changed by one per repetition period. In a conventional altimeter, the average phase of vector $K A$ is $\omega_{c} \tau$, as in figure 1 . If a frequency shift of $f_{d}$ cycles is introduced by FS, $K A$ rotates slowly with an angular velocity $\omega_{d}$, as shown in figure 7 . Hence, the average position of $K A$ is now time-dependent. If the modulating frequency is $f_{r}, K A$, while rotating slowly at a rate $f_{d}$, swings back and forth through $\gamma$ at a rate $f_{r}$.

\section{Methods of Obtaining Nonquantized Distance Information}

Three methods of obtaining nonquantized distance information have been investigated and are described below.

\subsection{Amplifier With a Uniform Frequency Characteristic}

If amplifier $\mathrm{A}$ in figure 6 has a uniform frequency characteristic, the signal fed into the counter $\mathrm{C}$ has the shape as shown in figure 7 . The counter produces one pulse for every positive-going zero-crossing. Hence, it is evident that the total count grows with increasing $\gamma$. It will be shown that a unique relation exists between the number of pulses per second and $\gamma$, that is, distance. The signal in figure 7 that is the detected signal can be represented by the following equation

$$
E_{c}=K A \cos \left(\theta+\omega_{d} t+\gamma \cos \omega_{r} t\right)=K A \cos \alpha .
$$

This equation follows directly from eq (3) with the assumption that $\omega_{c} \tau$ represents the constant phase angle $\theta$ and that the transit time $\tau$ is very small com-

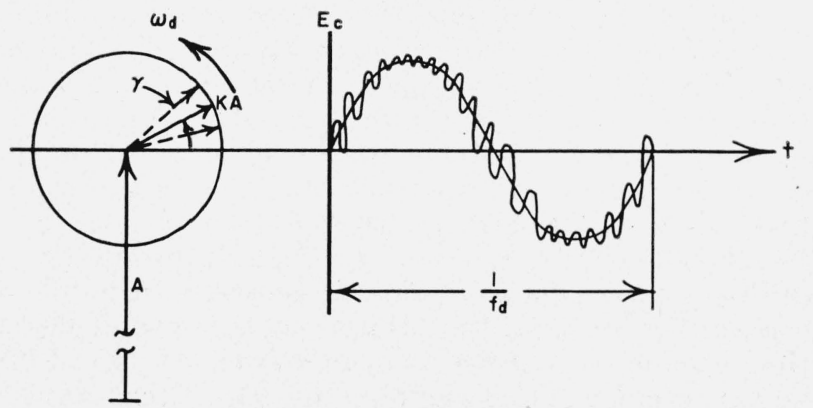

FiguRE 7. Detected signal of nonquantized altimeter. pared to $t$. A zero-crossing is obtained whenever $E_{c}$ becomes zero. Hence, the time instants for zerocrossings can be derived from the transcendental equation

$$
\cos \left(\theta+\omega_{d} t+\gamma \cos \omega_{\tau} t\right)=\cos \alpha=0 .
$$

This equation is solved graphically as shown in figure 8. Zero-crossings occur whenever $\alpha$ becomes $\pm(2 n+1) \pi / 2$ for integral values of $n$. It is evident that a count of 2 per repetition period is obtained for $\gamma=\pi$ (2 times the critical distance). A count of 1 is produced by $\gamma=\pi / 2$.

Figure 9 is similar to figure 8 . The time, however, is extended over many cycles of frequency $f_{d}$ because the counter used measures the average count over a long time. During each of the intervals $A B$, the oscillations of $\alpha$ are crossing the values $(2 n+1) \pi / 2$ The distance $\overline{A B}$ is proportional to the number of counts if it is assumed that $f_{r} \gg f_{d}$. Now, it can be seen that the total count over time $T$ is proportional to the sum of all lines $\overline{A B}$.

$$
\overline{A B}=\frac{2 \gamma}{\tan \epsilon} .
$$

Since $\tan \epsilon=\omega_{d}$, then

$$
\overline{A B}=\frac{2 \gamma}{\omega_{d}} .
$$

Because $\overline{A B}$ is proportional to $\gamma$, the total count is also linearly related to $\gamma$. This is strictly true as long as the observation time is large compared with the period of frequency $f_{d}$. In this case, the end effects occurring at time 0 and $T$ can be neglected. It can be seen from figure 9 that the count is proportional
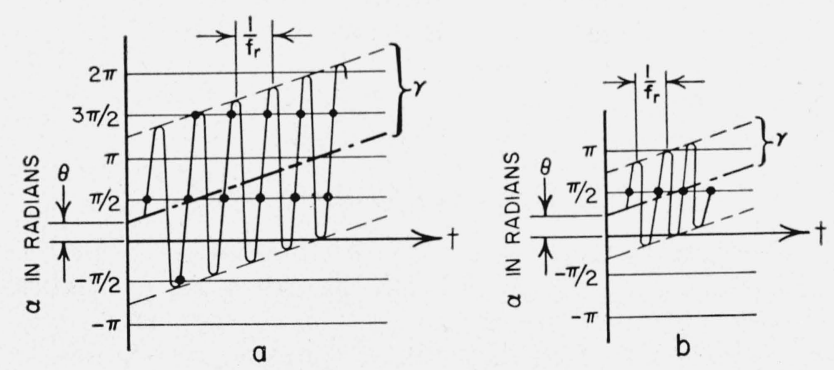

Figure 8. Zero-crossings from $\alpha$ verus $t$ curve.

o, zero-crossing of $E_{c}$
a, when $\gamma=\pi ;$ b, when $\gamma=\pi / 2$.

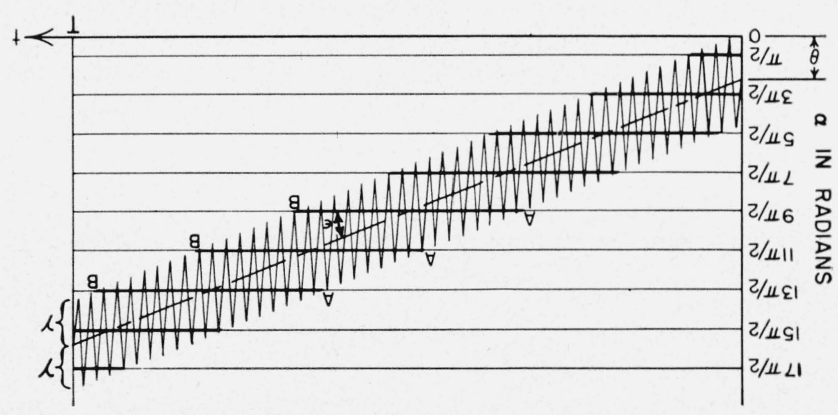

Figure 9. Location of zero-crossings over a long time interval. 


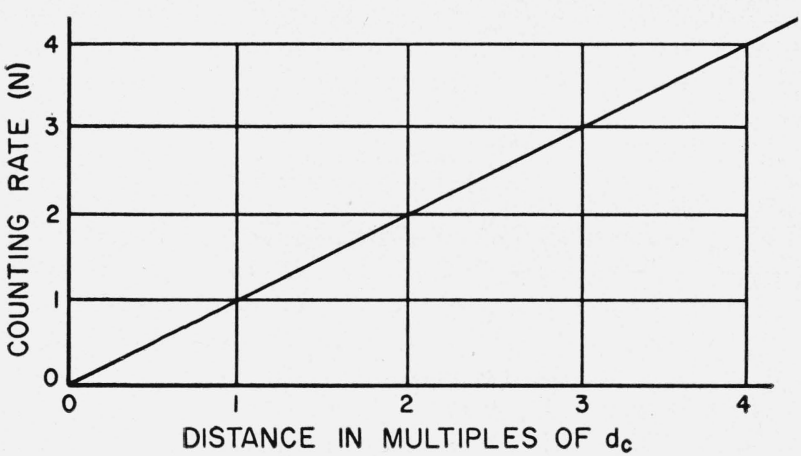

FIgURE 10. Theoretical output of nonquantized altimeter with flat amplifier.

to distance with one negligible error: for zero distance a count of $f_{d}$ pulses per second is obtained due to the presence of $f_{d}$. For $f_{d}=100 \mathrm{cps}$ and $f_{r}=10,000 \mathrm{cps}$, this error is only 1 percent for the critical distance. For higher values of $\gamma$, the error decreases proportionally. A plot of counts versus distance is shown in figure 10. If this curve is compared with the graph of figure 5, the advantages of the new system become evident.

If the frequency shifter FS would produce only energy at $f_{c}+f_{d}$, the linear relationship between distance and counts as shown in figure 10 could be maintained under all conditions. Unfortunately, practical frequency shifters also pass a small amount of energy at the frequency $f_{c}$ and if the shifter is not perfectly balanced, energy at the frequency $f_{c}-f_{d}$. This condition corresponds to that of a carrier with one or two sidebands separated by $f_{d}$. If this mixture is fed into the rectifier $\mathrm{R}_{e}$, a signal at frequency $f_{d}$ is registered by the meter as long as the amplitude of the desired signal does not exceed the amplitude of the envelope of the undesired mixture. This is similar to the capturing effect in conventional FM receivers and is due to the action of the limiter which is incorporated in the counter C.

\subsection{Amplifier With $f_{d}$ Filter}

For low-altitude use, the system as described is still practicable because of the large signal available. If, however, the signal level is small, the frequency characteristic of the amplifier $\mathrm{A}$ has to be shaped in such a way that energy at the frequency $f_{d}$ is eliminated from the counter. The conditions with an $f_{d}$ filter are shown in figure 11 . Whereas a count of 1 per repetition cycle was obtained only during a short time without the filter, a count of 1 is now produced during the larger part of a cycle of the wave at

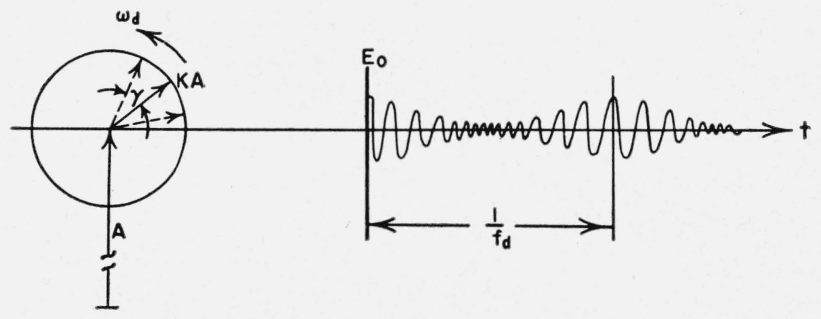

Figure 11. Signal output of $f_{d}$ filter. frequency $f_{d}$ so that a count versus distance curve different from the one shown in figure 10 is expected. Figure 11 is obtained by subtracting the $f_{d}$ component from the wave form in figure 7 . An alternate point of view is to consider that each wave formation per repetition-rate cycle is referred to its own average value.

Equation (4) expressed the signal fed into the amplifier. A Fourier expansion leads to

$$
\begin{aligned}
E_{c}= & K A\left\{J_{0}(\gamma) \cos \left[\theta+\omega_{d} t\right]-\right. \\
& J_{1}(\boldsymbol{\gamma})\left[\sin \left[\theta+\left(\omega_{d}+\omega_{r}\right) t\right]-\sin \left[\theta+\left(\omega_{d}-\omega_{r}\right) t\right]\right]- \\
& \left.J_{2}(\gamma)\left[\cos \left[\theta+\left(\omega_{d}+2 \omega_{r}\right) t\right]-\cos \left[\theta+\left(\omega_{d}-2 \omega_{r}\right) t\right]\right]-\ldots\right\}
\end{aligned}
$$

If a filter incorporated in the amplifier removes the energy at the frequency $f_{d}$, the amplifier output is represented by

$E_{0}=K A\left\{\cos \left[\theta+\omega_{d} t+\gamma \cos \omega_{\tau} t\right]-J_{0}(\gamma) \cos \left(\theta+\omega_{d} t\right)\right\}$.

With the filter, zero-crossings are obtained for $\mathrm{E}_{0}=0$. Whereas it was possible to solve eq (4) graphically, the number of zero-crossings for eq (6) has to be determined point by point for many average positions of vector $K A$ for each particular value of $\gamma$. After this has been done for a full revolution, the arithmetic mean of the individual numbers is determined, and constitutes the reading that an averaging counter would show. In other words, instead of considering a continouus rotation of $K A$ with an angular velocity $\omega_{d}$, it is assumed that the vector is periodically arrested and that it swings back and forth by the angle $\gamma$. This procedure is justified because the frequency $f_{d}$ is very small compared to $f_{r}$.

In figure 12 , voltage versus time is plotted for the equation

$$
E_{c}=K A \cos \left(\theta+\boldsymbol{\gamma} \sin \omega_{r} \mathrm{t}\right) .
$$

In the upper left part of the figure, the vector $K A$ is shown in its average position $\theta_{1}$ which is equal to $\theta+\omega_{d} t$. It is displaced from the vertical by $\theta_{1}=10^{\circ}$ and swings through a $\gamma$ of $\pm 270^{\circ}$ with sinusoidal velocity distribution. 'The lower part of the figure shows $\boldsymbol{\gamma} \sin \omega_{r} t$. Combining components, the complete wave is obtained as shown in the upper right of figure 12 .

If a uniform frequency characteristic were employed, that is, if no rejection filter for $f_{d}$ were used, the number of counts per repetition period would be determined by the crossings between the curve and the abscissa. With the filter, however, the number of counts per repetition period is determined by the crossings between the curve and its average value (over each variation per repetition-rate cycle). It is possible to obtain the new average value for every individual average position of vector $K A$ by cumbersome integration so that the areas above and below the zero line are identical. A more elegant procedure, however, is feasible if the fact that the filter removes the $J_{0}$ term of eq (6) from the curve in figure 12 is 


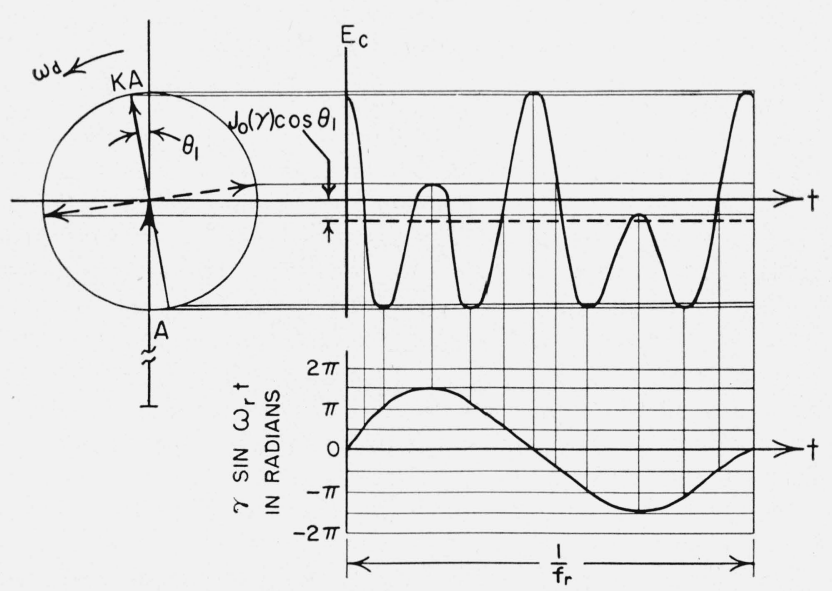

Figure 12. Zero-crossings of nonquantized altimeter with $f_{d}$ filter.

$\gamma=270^{\circ}=4.72 ; J_{0}(\gamma)=-0.27 ; \theta_{1}=10^{\circ}$;

$\cos \theta_{1}=0.98 ; J_{0}(\gamma) \cos \theta_{1}=-0.265$

taken into consideration. The slowly varying $J_{0}$ term is the average value during the repetition cycle. Accordingly, $J_{0}(\gamma)$ was obtained from tables, and $J_{0}(\gamma) \cos \theta_{1}$ was plotted in figure 12 as a horizontal line. The new count is obtained by determining the crossings between the curve and the dashed line. By inspection it can be seen that the count for the specific $\theta$ and $\gamma$ shown is higher if a filter is employed. The angle $\theta_{1}$ is varied by discrete steps, and the count is determined for each step. As mentioned before, the total count is the arithmetic mean of all the individual counts. This procedure is followed for many values of $\gamma$ so that, in the end, the count versus distance function is obtained.

Furthermore, it is clear that the number of counts with and without filter are identical for all values of $\gamma$ for which $J_{0}(\gamma)$ equals zero. The procedure leading to figure 12 was repeated for other phase angles in $10^{\circ}$ intervals and for values of $\gamma$ in $15^{\circ}$ intervals. A count versus distance curve was obtained as shown in figure 13. The linear curve corresponding to the flat amplifier is also shown, and it can be seen that the counts are identical for values of $\gamma=138^{\circ}$ and $315^{\circ}$, for which $J_{0}(\gamma)$ is zero. In addition, because of symmetry, the counts are also identical for $\gamma=180^{\circ}$ and $360^{\circ}$, etc. For higher values of $\gamma$, the two curves become more and more similar, the reason being that $J_{0}(\gamma)$ becomes progressively smaller. The height-indicating meter must be equipped with a dial calibrated according to the curve in figure 13 . The necessity for such a dial calibration is not a serious disadvantage because the deviation from linearity is small beyond the critical distance. As compared with standard altimeters, the only additional component is the frequency shifter, which is inserted between power oscillator and mixer.

\subsection{Amplifier With Peaked Frequency Characteristic}

A method of obtaining a linear count versus distance that suppresses interference from the leakage

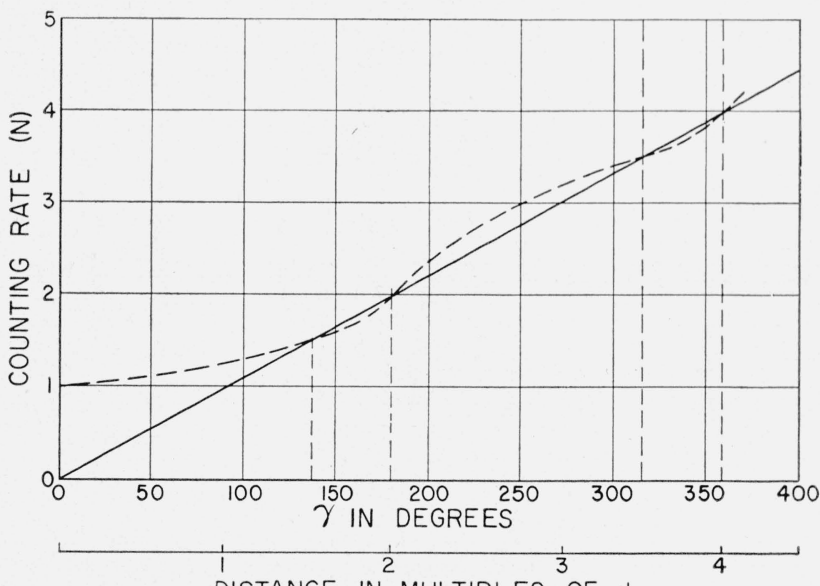

DISTANCE IN MULTIPLES OF $d_{c}$

FIGURE 13. Theoretical output of nonquantized altimeter with $f_{d}$ filter. $498^{\circ}$, etc., $f_{d} \ll f_{r}$, with $f_{d}$ filter;

signal at frequency $f_{c}$ employs an amplifier having a response proportional to frequency and a slope of 6 $\mathrm{db}$ per octave (fig. 14). Since leakage signals produce an $f_{d}$-beat output at the mixer, and since $f_{d} \ll f_{r}$, the amplifier suppresses this interference signal by at least $f_{r} / f_{d}$. The amplifier output voltage will be the derivative of the input voltage. The effect of such an amplifier on the mixer output that is given by eq (4), $E_{c}=K A \cos \left(\theta+\omega_{d} t+\gamma \cos \omega_{\tau} t\right)$ will be

$E_{0}=\frac{d E_{c}}{d t}=-K A\left(\omega_{d}-\gamma \omega_{r} \sin \omega_{r} t\right) \sin \left(\theta+\omega_{d} t+\gamma \cos \omega_{r} t\right)$.

Counts will be obtained, as before, for each positivegoing zero-crossing. Zeros of eq (7) will occur where either the factor $F_{2}=\sin \left(\theta+\omega_{d} t+\gamma \cos \omega_{r} t\right)$ is zero or when factor $F_{1}=\left[\omega_{r}-\gamma \omega_{r} \sin \omega_{r} t\right]$ is zero. The former factor produces a count proportional to distance, as shown in figure 10 . When the second factor $F_{1}$ is plotted (fig. 15), one positive-going zerocrossing per $1 / f_{r}$ sec or $f_{r}$ counts per second occur. The only effect of changing $\gamma$ is to increase the amplitude of the sine wave, of figure 15 . Since $\omega_{r} \gg$ $\omega_{d}, \gamma \omega_{r}>\omega_{d}$ except for extremely small $\gamma$. As a result, the counter versus distance characteristic will be as shown in figure 16 .

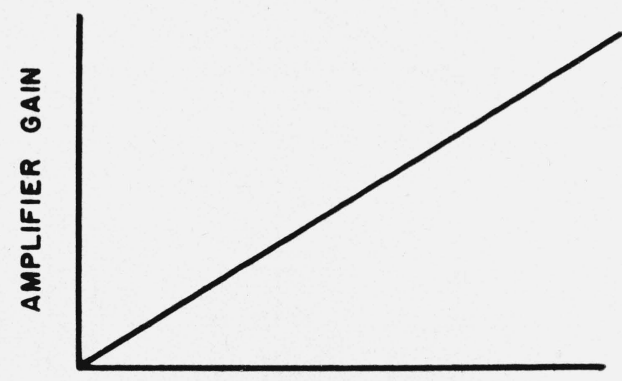

FREQUENCY

FIGURE 14. Frequency characteristic of peaked amplifier. 


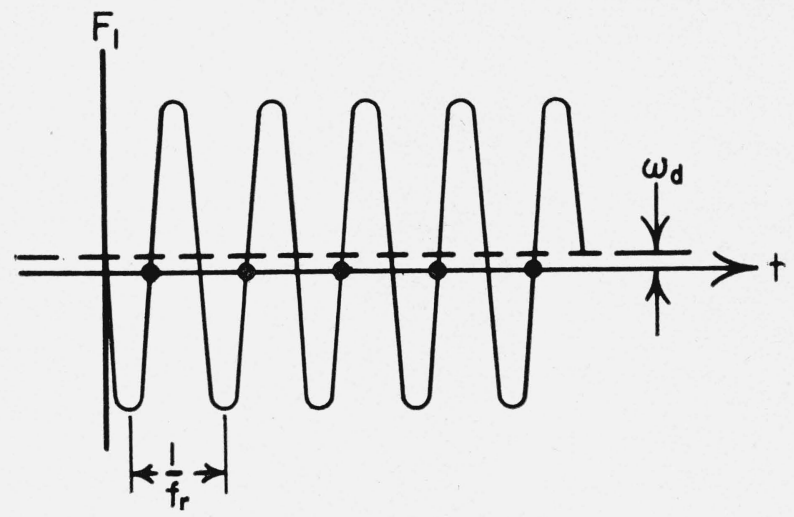

FIGURE 15. Zero-crossing introduced $\left(\omega_{d}-\gamma \omega_{r} \sin \omega_{r} t\right)$ of equation (7).

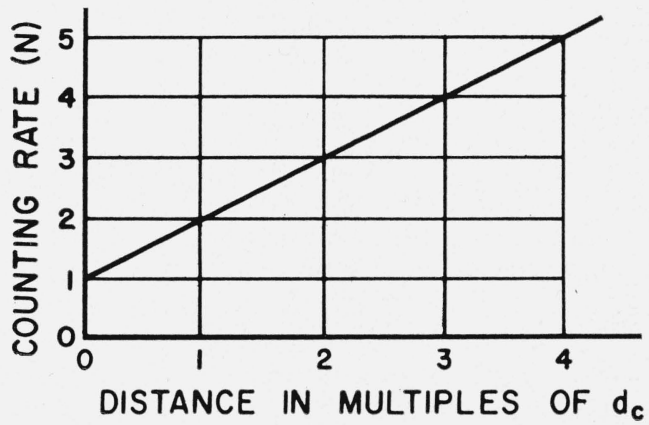

FIGURE 16. Theoretical output of nonquantized altimeter with peaked amplifier.

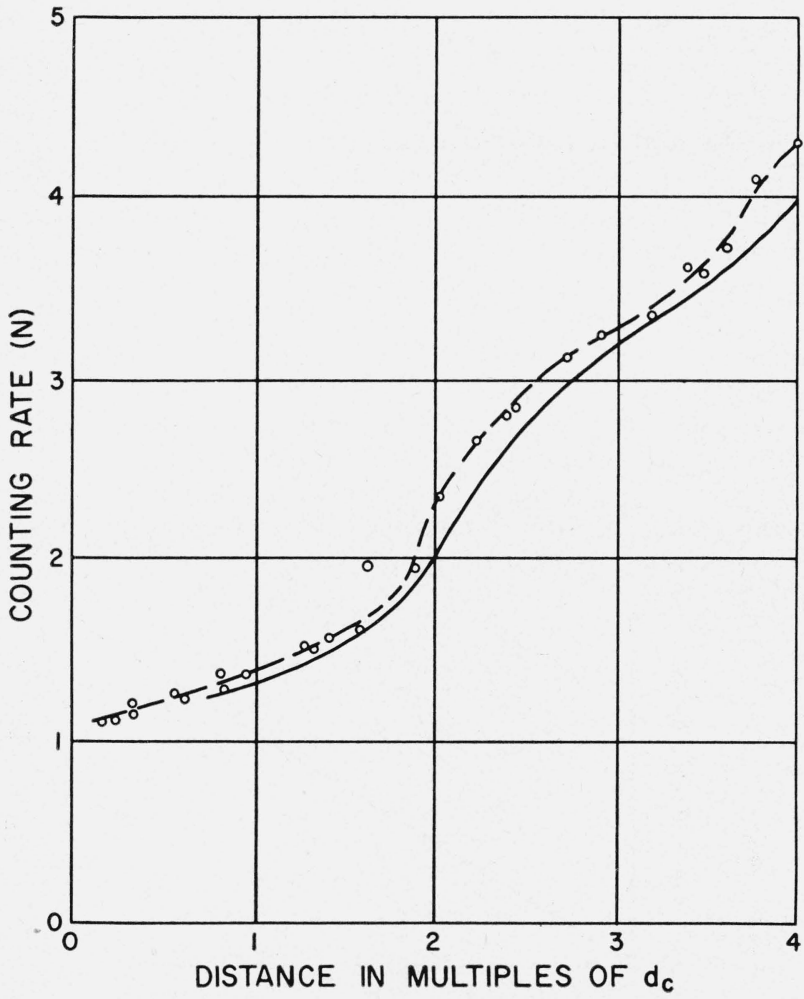

FIGURE 17. Measured output of nonquantized altimeter with $f_{d}$ filter.

- $-\ldots$, Experimental; $\_-$, theoretical

\section{Experimental Results}

Results of experiments using the three types of frequency response characteristics are shown in figures 17 and 18 . In figure 17, the $f_{d}$ filter was used. The experimental points were obtained from several test runs, using different klystron deviations (different values of $\beta$ ) after reducing the distance in terms of appropriate critical distances for each run. The agreement with the theoretical curve reproduced from figure 17 is clearly shown. Figure 18 shows a set of runs, all having the same $\beta$, but using the different networks. These curves are in agreement with theory in all respects.

Summarizing, there are three methods of obtaining nonquantized distance information:

(1) If an amplifier with uniform amplitude characteristic is employed, the count is proportional to distance, as shown in figure 10. The method is usable only where the return signal overrides the oscillator signal leaking through the frequency shifter.

(2) If an amplifier with a rejection filter for $f_{d}$ is employed, the count follows a curve as shown in figure 13.

(3) If an amplifier with a frequency response proportional to frequency is used, the count follows the linear relation shown in figure 16 . Whereas method (1) is practicable only as shown above, methods (2) and (3) are usable for any distance within the range of conventional altimeters.

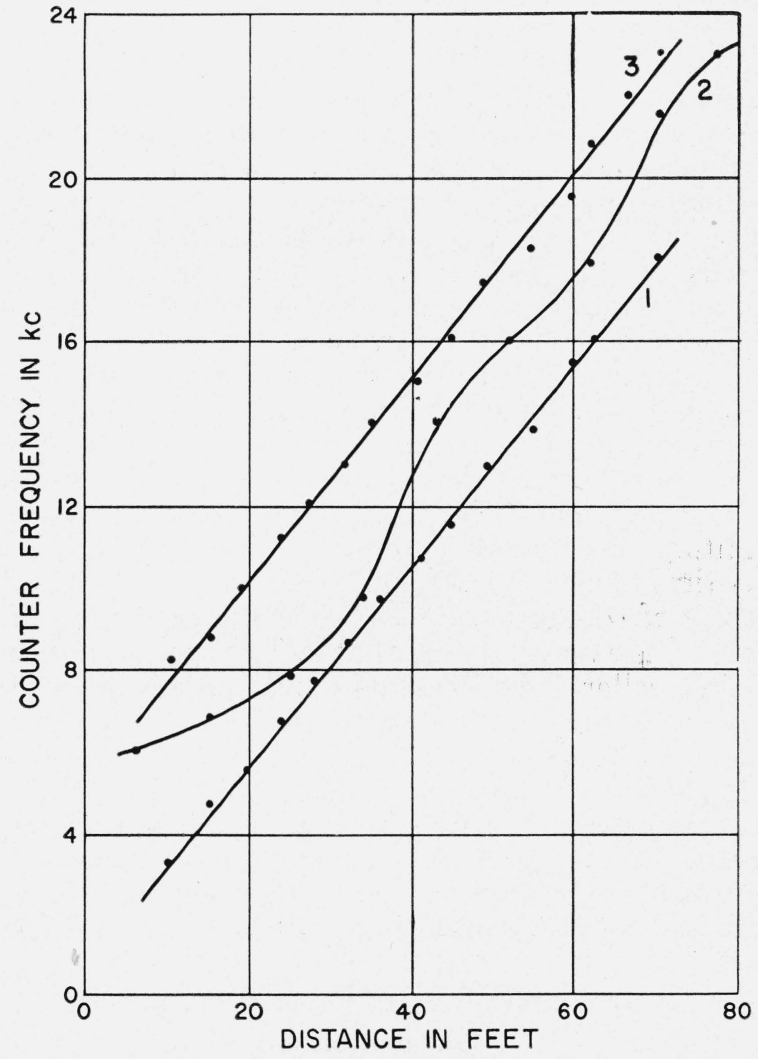

FIGURE 18. Measured output of nonquantized altimeter versus distance.

$f_{r}=5 \mathrm{kc} ; f_{d}=100 \mathrm{cps}$

1, Flat amplifier; 2, flat amplifier with $f_{d}$ filter; 3 , peaked response amplifier. 


\section{Appendix}

\subsection{Frequency Shifter}

The frequency shifter is used for producing a signal with a frequency displaced from the frequency of the transmitted energy by a constant amount. This alternating voltage, which serves as the local signal for the mixer, has to follow the frequency excursions of the power oscillator accurately without vime delay. Although it is possible to use, for this purpose, a second oscillator controlled by a fast-acting servo loop in such a way that a constant frequency difference is maintained, a system of this kind should be avoided because of its complexicy and because of the wide frequency-response characteristic required for the servo amplifier. A better solution is the use of a mechanical, or electrical, continuous phase shifter inserted between the power oscillator and the mixer. For frequencies up to 300 megacycles, mechanical single-sideband modulators with capacitive-type or goniometer-type phase shifters can be used. ${ }^{1}$ For microwave-frequency applications, the two following devices can be employed.

(1) Continuous phase shifter employing circular polarization: ${ }^{2}$ This device is especially suited for microwaves. It consists of three wave-guide sections as shown in figure 19. $\mathrm{A}$ is at rest and contains a quarter-wave plate so that an entering plane wave emerges circularly polarized into the plane $\mathrm{P}$. $\mathrm{B}$ is a physically rotating circular wave guide, containing a half-wave plate so that the direction of rotation of the $E$ vector is reversed. $C$ is at rest, like $A$, and also contains a quarter-wave plate so that a circularly polarized wave entering at $\mathrm{P}_{2}$ emerges as a plane wave.

A plane-polarized wave is fed into section $\mathrm{A}$ and emerges circularly polarized in, say, a clockwise direction. Hence, the $E$ vector in the plane $\mathrm{P}_{1}$ performs $f$ rotations per second, $f$ being the carrier frequency of the incoming energy. If an observer were located in B and if B rotates counterclockwise, the observer will see a frequency $f+n, n$ being the number of revolutions per second of section $B$. Due to the action of the half-wave plate, the observer will see $f+n$ counterclockwise rotations of the $E$ vector in the plane $\mathrm{P}_{2}$. Since $\mathrm{B}$ itself rotates counterclockwise, the $E$ vector in $\mathrm{P}_{2}$, as seen from the section $\mathrm{C}$, rotates $f+2 n$ times a second. Energy with a frequency $f+2 n$, therefore, emerges from $\mathrm{C}$.

If the device would be designed without the half-wave plate in $\mathrm{B}$, the observer would still see $f+n$ rotations in this section. As seen from $\mathrm{C}$, however, $f+n-n$ rotations would be observed so that outcoming and incoming frequencies would be identical.

(2) Single-sideband generator, employing gyrators: ${ }^{3}$ Three waves of the same frequency $f_{c}$ and with phases displaced by $120^{\circ}$ are superimposed. The waves are amplitude-modulated with envelopes of the frequency $f_{d}$, and the phases of the envelopes are also displaced by $120^{\circ}$. In figure 20 , the

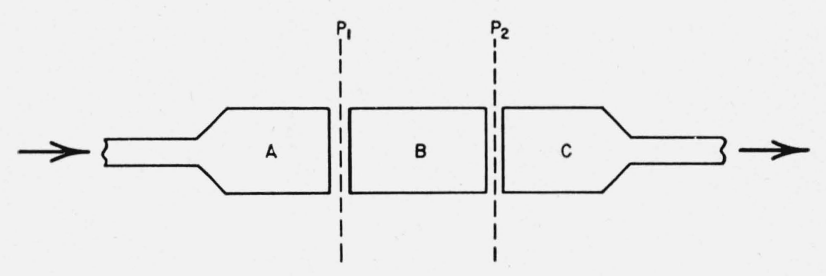

Figure 19. Continuous phase shifter.

\footnotetext{
${ }^{1}$ H. R. Holloway and H. C. Harris, Precision frequency generators using singlesideband suppressed-carrier modulators, Sylvania Technologist, 4,3, 64-67 (July 1951)

2 A. G. Fox, An adjustable waveguide phase changer, Proc. IR E 35, 1489-1498 (December 1947).
}

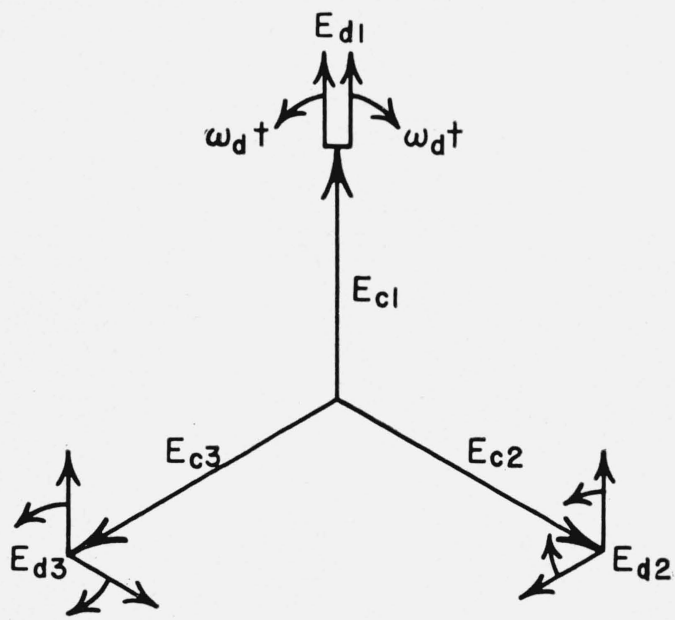

Figure 20. Vector diagram, three amplitudemodulated carriers.

three carrier waves are represented by the vectors $E_{c 1}, E_{c 2}$, and $E_{c 3}$. Each of the envelopes $E_{d 1}, E_{d 2}$, and $E_{d 3}$ is represented by two sidebands, one rotating clockwise and the other counterclockwise. At the instant shown, it is assumed that $E_{c 1}$ has reached its maximum amplitude, whereas the envelopes of $E_{c 2}$ and $E_{c 3}$ are delayed by $120^{\circ}$ and $240^{\circ}$, respectively. As the result of the superposition of all nine signals, it can readily be seen that $E_{c 1}, E_{c 2}$ and $E_{c 3}$ cancel each other. Furthermore, there is a cancellation of the clockwise components of $E_{d 1}, E_{d 2}$ and $E_{d 3}$. The counterclockwise components, however, add so that a resultant voltage of $3 E_{d}$ is obtained with a frequency of $f_{c}-f_{d}$ cps.

Until recently, systems of this kind could not be realized at microwave frequencies because amplitude modulation without simultaneous frequency modulation was not possible. The microwave gyrator, ${ }^{4,5}$ which is a new circuit element, has been developed for amplitude-modulation purposes. The gyrator rotates the plane of polarization of an electromagnetic wave in accordance with Faraday's principle. A ferromagnetic material, such as Ferramic $A$, is inserted in circular waveguide and a steady magnetic field is applied in the direction of propagation. Polarized microwaves, when propagated through the magnetized Ferramic material, exhibit appreciable Faraday rotation. With rectangular waveguide at the input of the gyrator at right angles to the wave guide at the output and with no current in the gyrator coil, power will not be transferred from the input to the output waveguide. When the gyrator coil is energized, the plane of polarization of the electromagnetic wave is rotated so that power is transferred. When an alternating voltage is applied to the coil, the microwave signal is amplitude-modulated at a rate dependent upon the frequency of the impressed voltage. Above a few cycles per second, conventional gyrators cannot be used as amplitude modulators because the metal waveguide acts as a shorted turn inside the coil and attenuates the alternating magnetic field. A new gyrator was designed for modulation frequencies of the order of 20 kilocycles. Instead of metal waveguide, a linen Bakelite form covered with silver paint was used.

${ }_{3}$ J. Cacheris and H. Dropkin, Single-sideband suppressed-carrier microwave modulator, NBS Report 17-49.

4 C. H. Luhrs, Correlation of the Faraday and Kerr magneto-optical effects in transmission-lines terms, Proc. IRE 40, 76-78 (January 1952).

5 C. L. Hogan, The microwave gyrator, Bell System Tech. J. 31, 1-31 (January $1953)$.

Washington, February 26, 1953. 